doi:10.1088/1741-4326/aac4e5

S. Äkäslompolo et al 2018 Nucl. Fusion 58082010

Author accepted manuscript

\title{
Modelling of NBI ion wall loads in the W7-X stellarator
}

S. Äkäslompolo ${ }^{1}$, M. Drevlak ${ }^{1}$, Y. Turkin ${ }^{1}$, S. Bozhenkov ${ }^{1}$, T. Jesche ${ }^{1}$, J. Kontula ${ }^{2}$, T. Kurki-Suonio ${ }^{2}$, R.C. Wolf ${ }^{1}$ and the W7-X team

${ }^{1}$ Max-Planck-Institut für Plasmaphysik, Wendelsteinstraße 1, 17491 Greifswald, Germany

${ }^{2}$ Department of Applied Physics, Aalto University, FI-00076 Aalto, Finland

E-mail: simppa.akaslompolo@alumni.aalto.fi

23 April 2018

\begin{abstract}
It is known that the NBI ion losses may pose a problem to the Wendelstein 7-X first wall, but the so-far utilized wall models had insufficient details for reproducing the locations and magnitude of the hot-spots. This study aims to exhaustively analyse the detailed wall loads in the reference magnetic configurations and in various plasma scenarios. The goal is to pinpoint the endangered plasma facing components and to calculate heat loads to them in preparation for the first NBI operations. This study can be used to prepare monitoring of the heat loads and paves the way to future search of improved configurations.
\end{abstract}

Keywords: stellarator, energetic particles, neutral beam injection, fast particle confinement Submitted to: Nucl. Fusion 


\section{Introduction}

Wendelstein 7-X (W7-X) is a stellarator with modular superconducting coils. It is the latest and largest of the helical axis advanced stellarator (HELIAS) line. The complex 3D shape of the coils was optimised i.a. to have "good $\alpha$-particle confinement in reactor extrapolation" [1. The W7-X magnets can produce different magnetic configurations with special properties e.g. moving the plasma horizontally or changing the edge rotational transform (iota, $\iota$ ).

The neutral beam injection (NBI) systems 2 are currently being installed in the machine, with two primary missions: to provide (ion) heating capability in addition to the existing electron cyclotron heating (ECRH) and to create a source of fast ions. Both missions are in support of demonstrating the W7-X optimisation. The field was optimised to minimise the radial drift of trapped particles through isodynamicity [3], which is expected to occur in the plasma core at high plasma pressure $(\langle\beta\rangle>4 \%)$. Thus, orbit losses were expected to be negligible [1]. Accordingly, the machine plasma facing component (PFC) design does not include dedicated protection against fast ion impacts. However, detailed numerical studies have shown the confinement of fast ions to be far from ideal $2,4,5,6,7,8,9$, especially in the initial NBI studies when the $\langle\beta\rangle$ is not expected to reach the values the field was optimized for. The losses are exacerbated by the nearly radial neutral beam injection geometry in W7-X. The initial pitch angles of the beam ions peak at about $17^{\circ}$ or $27^{\circ}$ from perpendicular, depending on the used ion source. The radial injection produces ions that are close to being trapped, so that many scatter to orbits that are reflected by the periodic toroidal magnetic well of W7-X. Moreover, a fraction of the ions scatter to "ripple trapped" or "superbanana" orbits: they are reflected between the modular coils and can drift quickly out of the plasma to the wall. Indeed, most hot spots on the PFCs are located at the intersection of the wall and the local magnetic wells, as illustrated by figure 1 .

If left uncontrolled, the heat and particle load due to the NBI ions may heat up and damage the PFCs, with stainless steel components being particularly vulnerable. However, even before damage, the PFCs may release enough impurities by outgasing and sputtering to dilute or even collapse the plasma.

The existing research 2, 4, 5, 6, 7, 8, 9 has already shown that the fast ion loads may pose a problem to the first wall, but the utilized wall models have insufficient details for reproducing the locations and magnitude of the hot-spots. This study aims to exhaustively analyse the detailed wall loads for pinpointing the endangered plasma facing components and to calculate heat loads to them in preparation for the first NBI operations. This study can be used to prepare monitoring of the heat loads and paves way to future search of improved configurations beyond the reference ones.

This study is composed of a number of parameter scans. The high mirror configuration near $\langle\beta\rangle=2 \%$ is chosen as the central point in the parameter space. The change in fast ion behaviour is studied by first choosing three plasma profiles at up to $\langle\beta\rangle=4 \%$ and testing all the magnetic configurations. Since the hotspot locations are primarily set by the magnetic field and the plasma facing components, it is possible to disentangle the magnetic configurations and the effect of the plasma. Thus, the magnetic field configuration is kept constant and the plasma profiles are modified at various combinations with $\langle\beta\rangle<2 \%$. This produces a comprehensive understanding on how the fast ion population can be affected while still limiting the number of degrees of freedom in each scan. The different reference magnetic configurations are studied in section 3. and the different plasma profiles are studied in section 4. The scans are preceded by an introduction of the used tools in section 2 and succeeded by a summary and presentation of further work in section 5 


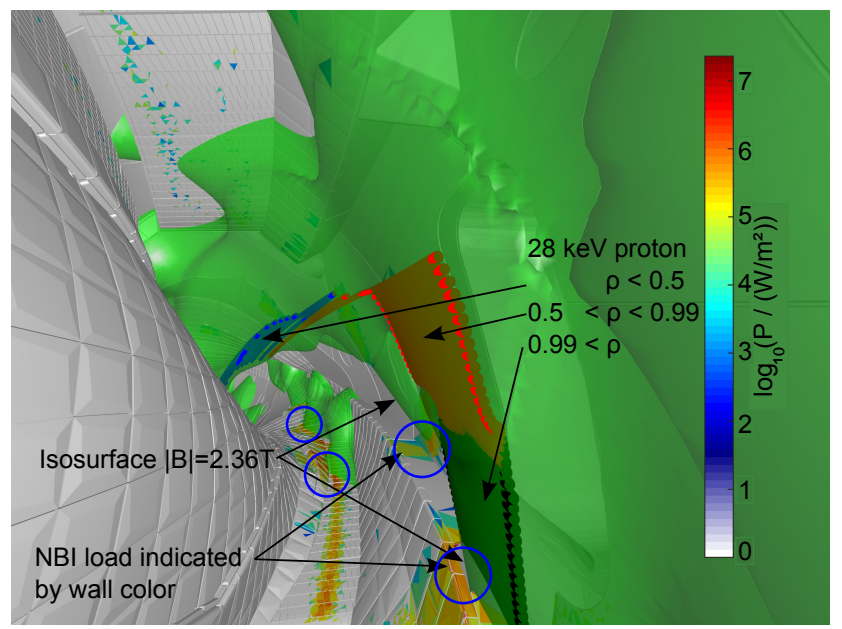

Figure 1. Perspective view into the plasma vessel illustrating the main wall load mechanism. The wall color indicates the wall load from the NBI ions in logarithmic scale. A $|\boldsymbol{B}|$ isosurface is drawn in translucent green to illustrate the local magnetic wells due to the modular coils. Fast ions trapped in such wells drift quickly to the wall due to the gradient-B drift $(\nabla B \times \boldsymbol{B})$. This is illustrated by following a $28 \mathrm{keV}$ proton collisionlessly while omitting the radial electric field. The proton trajectory is colored according to the minor radius ( $\rho$, square root of the normalized toroidal flux) of the path. The $|\boldsymbol{B}|$ isosurface value is chosen to match the reflection magnetic field $(E / \mu)$ of the ion, $2.36 \mathrm{~T}$. Hence, the ion is toroidally reflected at the isosurface and remains in the well and finally drifts to the wall. Similarly, most of the hot spots on the wall are located where the wall intersects the wells (blue circles). The simulation is for the reversed field W7-X standard configuration $\langle\beta\rangle=2 \%$. Here the ions drift downwards, while in a forward field case the hot spots would be located at the stellarator symmetric 10 top intersection areas.

\section{Description of the computational methods}

The study presented here uses W7-X standard tools for magnetic field and plasma profile calculations as well as the ASCOT suite of codes, in particular ASCOT4 [1] and BBNBI [12], for fast ion modelling. The workflow is presented in figure 2 and starts by choosing the magnetic configuration of interest, i.e. the currents in the 7 main coil systems of W7-X. Next, the VMEC code [13] is used to calculate the MHD equilibrium with some reasonable plasma pressure profile and vanishing toroidal current.

The plasma profiles that correspond to the particular equilibria and heating scheme are obtained by modeling using NTSS code [14 that uses transport coefficients from DKES [15]. The ECR plasma heating is modeled by the TRAVIS code [16. The transport model is chosen to be mainly neoclassical with additional anomalous-like transport with the value $1-2 \mathrm{~m}^{2} / \mathrm{s}$ at the plasma edge region. In simulations the density profile is fixed, while the ion and electron temperatures together with the ambipolar radial electric field are determined self-consistently. The assumption of mainly neoclassical transport leads

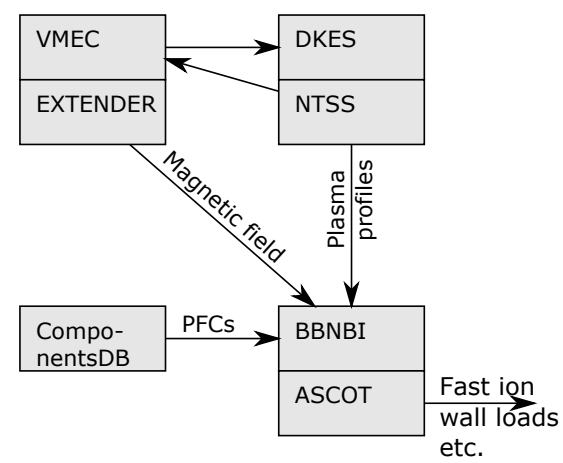

Figure 2. Flow of data from tool to tool. The VMECDKES/NTSS loop was iterated until the plasma pressure from NTSS was consistent with equilibrium from VMEC.

to an upper limit for the temperatures.

In certain cases, the resulting plasma pressure is fed back to VMEC and the cycle is iterated until it converges to self-consistent profiles and equilibrium. In other cases, the effect of a single variable, such as the magnetic configuration, is studied, and all other parameters remain constant regardless of changes in the studied variable. Here, the self-consistent magnetic and electric fields serve only as a starting point for acquiring semi-realistic profiles. This requires relaxation of the self-consistency but pays off in simpler numerical experiments.

When the calculation of the profiles and equilibrium is complete, they are exported to ASCOT suite of codes using the EXTENDER [17] code, which also calculates the magnetic field outside the VMEC computation domain, i.e. outside the plasma.

The neutral beam ionization code BBNBI 12 . is then used to model the NBI ionisation. Half of the designed NBI sources [18, (Q3, Q4, Q7 and Q8), are being installed for the first NBI experiments projected for the year 2018. Sources Q3 and Q4 are in the injector NI20 (port K20) and sources Q7 and Q8 are positioned stellarator-symmetrically in injector NI21 (port K21). The velocities of ions from Q3 and Q7 are more perpendicular than Q4 and Q8. Each pair (Q3+Q7 or Q4+Q8) produces a stellaratorantisymmetric velocity distribution. In BBNBI, these four sources were set to inject hydrogen, with the nominal power: $1.7 \mathrm{MW}$, each. BBNBI uses a detailed model of the NBI injectors including 774 beamlets per source. The maximum particle energy for hydrogen injection is $55 \mathrm{keV}$ and the realistic fractions of $1 / 2$ and $1 / 3$ energy particles are included: $39 \%$ and $28 \%$, respectively. The results are found to be in good agreement with the NBI model within NTSS.

The time evolution of an ensemble of a million markers representing the ionized neutral beam particles are modelled with the ASCOT4 [11] code un- 
til they either collide with the 3D wall or are slowed down close to the local thermal energy. ASCOT4 interpolates the magnetic field with splines from a regular cylindrical grid. Within the plasma ASCOT4 follows guiding centres, and switches to resolving the full gyro-motion before the last closed flux surface (LCFS). Monte Carlo collision operators model the energy and pitch collisions with the static proton-electron plasma background defined by the input profiles. The detailed $3 \mathrm{D}$ wall (figure 3) is acquired from CAD models by exporting them as triangular surface meshes with $\sim 4$ million triangles [19]. The wall load is calculated simply by summing the contribution of all markers hitting a particular wall triangle and dividing by the triangle surface area. The triangles have a wide distribution of areas from $1 \mathrm{~mm}^{2}$ to $0.2 \mathrm{~m}^{2}$ with mean and median of $375 \mathrm{~mm}^{2}$ and $20 \mathrm{~mm}^{2}$, respectively.

The charge exchange $(\mathrm{CX})$ losses of the beam ions have been omitted in the current study. This is due to only partial implementation of the relevant atomic processes in the current version of ASCOT4 and also due to a large uncertainty in the neutral density in the plasma. The CX reactions are expected to reduce the peak load by neutralizing a small fraction of the particles on their way from the plasma to the hotspot. The resulting neutrals will be spread onto a large area on the wall. On the other hand, the CX losses are expected to increase fast ion transport at the edge leading potentially to more particles in the coil ripple and increased losses.

\section{Magnetic field configuration scan with 3 plasma profiles each}

The W7-X magnetic field is produced by 70 superconducting coils of 7 different shapes. The ten coils of each shape are connected in series, and each circuit has an independent power supply. Variation of the relative current in the coils gives rise to a six-dimensional magnetic configuration space [20]. The shape of the coils was optimised so that the enhanced confinement and other advanced features of the magnetic field are at least partially present in the 9 reference configurations listed in table 1 and described in 21, 22. The high mirror (HM) configuration is the best optimised case, where e.g. the fast ion confinement is expected to be at its peak, while the standard configuration (STD) is the "median configuration" at the centre of the configuration space. The low mirror (LM) configuration is closest to a classical stellarator configuration. The plasma can also be moved inward and outward to produce the inward shifted (IS) and outward shifted (OS) configurations. Wendelstein 7 -X has island divertors, which limit the edge rotational transform $(\iota)$ values to low order rationals with 5 in the numerator. For most configurations the edge iota approaches $5 / 5$, but for low iota (LI) and high iota (HI) configurations it approaches $5 / 6$ and $5 / 4$, respectively. For the low shear (LS) configuration, the $\iota$-profile is particularly flat.

In the current study, eight of the reference configurations are studied using the magnetic and plasma backgrounds originally created for 44 using methods described in section 2. Self-consistent magnetic field and plasma profiles were calculated for the standard configuration at $\langle\beta\rangle=2 \%$. Two more plasma profiles were generated to study the effect of increased plasma pressure by simply doubling the temperature $(2 \mathrm{xT})$ or density $(2 \mathrm{xn})$ of the selfconsistent profiles to achieve $\langle\beta\rangle=4 \%$, while the radial electric field was left unmodified. The doubling of the density increases the fast ion collisionality while doubling the temperature decreases it. As figure 4 shows, the density is relatively high, especially in the doubled density case. Accordingly, the radial electric
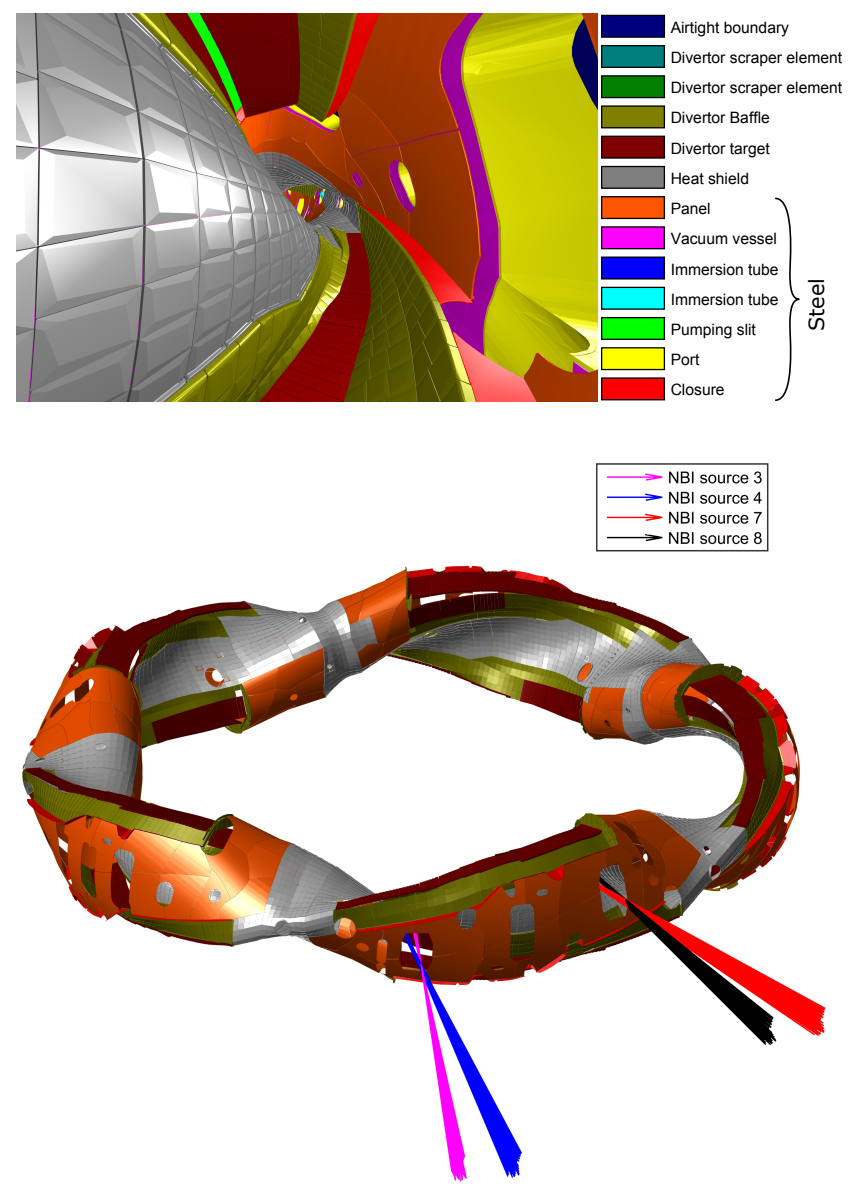

Figure 3. The wall components. The upper fiure shows internal view of the vacuum vessel while the lower figure shows the most inportant wall elements from the outside as well as the four modelled NBI sources. The divertor and the heat shield are mainly carbon components. The elements from panel to closure are predominantly stainless steel. The airtight boundary is a numerical construct only. 
Table 1. The eight magnetic configurations of W7-X 21,22 studied here. The ninth configuration, "Limiter", was not part of the study [4] and was excluded here as well.

\begin{tabular}{l|cl} 
& Name & Linestyle \\
\hline HM & High mirror & \\
STD & Standard case & \\
LM & Low mirror & \\
OS & Outward shifted \\
IS & Inward shifted & \\
LS & Low shear & \\
HI & High $\iota$ & \\
LI & Low $\iota$ &
\end{tabular}

field indicates ion root confinement throughout. These plasma profiles were then used to calculate magnetic fields in all 8 magnetic configurations, making a total of 24 cases. The magnetic field for $2 \mathrm{xT}$ and $2 \mathrm{xn}$ is identical, because the magnetic field calculation uses only the plasma pressure as input.

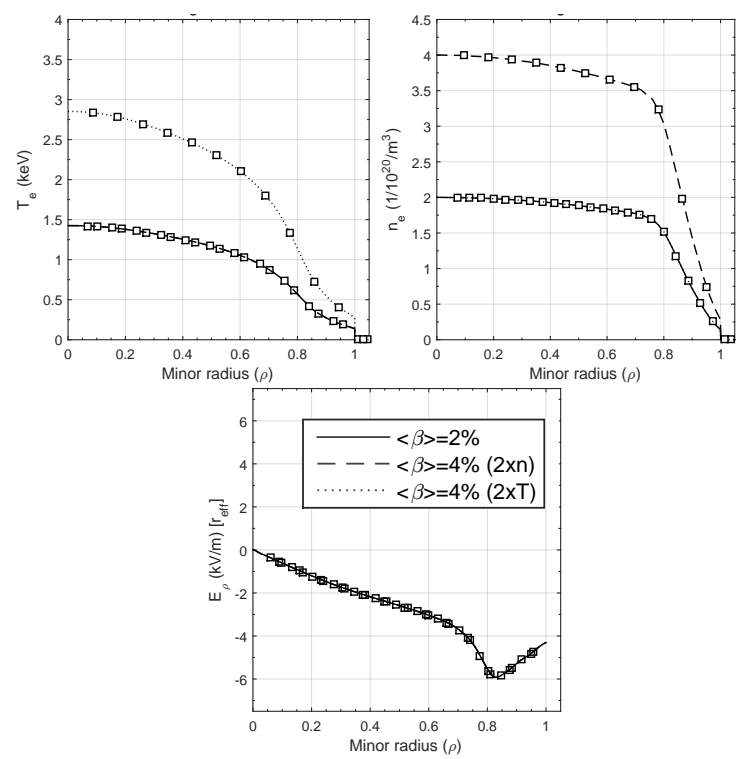

Figure 4. The plasma profiles for the magnetic configuration scan. The ion temperature is identical to the electron temperature $T_{e}$. The plasma is assumed to be pure protonelectron plasma. Identical radial electric field is assumed for all profiles.

\subsection{Configuration Scan Results}

The high mirror configuration produces the lowest NBI ion losses to the wall. This was learned through a series of simulations, each starting by first generating a million NBI ionisation locations with BBNBI for each case. The upper lines in figure 5 indicate that the doubling of density moves the deposition outwards while there is only minor variation between the magnetic configurations. The ASCOT4 code then calculated the slowing down distribution and wall loads of fast ions. For all cases, the bulk of the wall losses originate from the edge of the plasma, but there are differences in the magnitude of the losses between configurations as illustrated by the lower group of lines in figure 5. Furthermore, in the lower collisionality plasmas the losses extend from deeper in the plasma, even though the confinement is expected to improve towards the magnetic axis.

Increasing the collisionality reduces losses by slowing the fast beam ions to the thermal energy before the ions have time to escape the plasma. Indeed, it is expected that several tens of percent of the fast ions would eventually drift out of the plasma, if not slowed down [5]. The loss fraction as a function of time (figure 6) illustrates this: the fraction of NBI ions lost to the wall increases linearly until the slowing down stops the process, at least in the simulations. (The particle simulation stops when the marker has slowed down to the local thermal energy or hits a wall. However, in reality the losses may continue also after the thermalization. The confinement of such thermal particles is, however, outside the scope of this work.) The variation in fast ion confinement in different configurations is remarkably large: core loss fractions can change by a factor of about $3 \cdots 6$ even within the same plasma scenario, with the variation increasing with collisionality.

Studying the density of fast ions in the plasma provides another view to the confinement properties of the plasma. The studied fast ion density profile is an integral over the whole velocity space, thus summing over the whole slowing down process. Figure 7 indicates that the high mirror configuration produces the highest fast ion density at mid-radius in doubled temperature $(2 \mathrm{xT})$, while the low mirror configuration does so at the core of the doubled density (2xn). Study of the loss fractions and slowing down times as a function of ionisation location, beam source, initial particle reflection magnetic field and initial energy in HM and LM configurations revealed only that the effect is more pronounced for the more radial sources (Q3 and Q7) than for the more tangential sources (Q4 and Q8). The underlying explanation could be a peculiarity of the collisional transport properties of the LM configuration. It may be due to the reduced mirror field in LM reducing the fraction of phase space populated by trapped particles. However, due to the very small fast ion density in the $2 \mathrm{xn}$ plasmas, there are no foreseen practical consequences from the numerically observed effect.

The fast ion density was found to be relatively insensitive to the magnitude of the radial electric field: doubled field strength increased the density by only $\sim 5 \%$. 



Figure 5. The NBI ionization distribution (filled markers), which was created by simply binning the ionization locations in minor radius. Accordingly, the panels have identical but arbitrary ordinate unit. The peak at the edge is an artifact of the minor radius coordinate: it is only defined within the plasma. Therefore, markers ionized outside the plasma are all collected to the single bin at $\rho=1$. The lines with hollow markers depict the particles that were lost to the wall. The black cross is at same level, both in this figure, and in figure 14 The line legend is in table 1
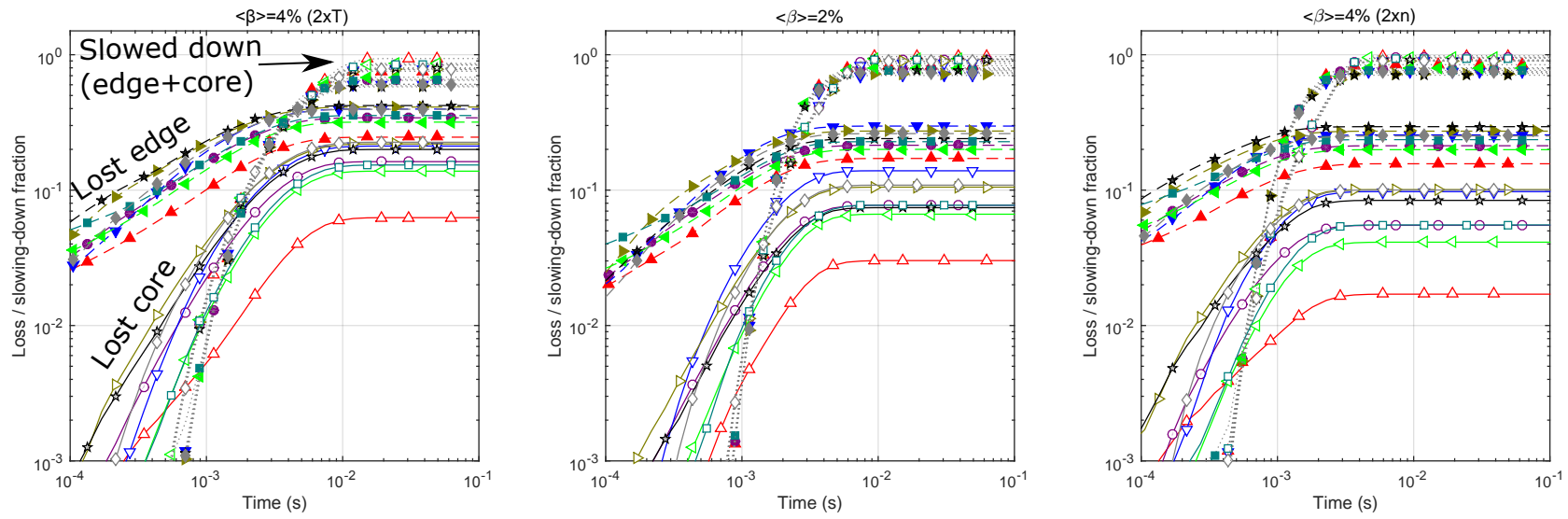

Figure 6. The fraction of particles lost to the wall (colored solid and dashed lines) and slowed down (gray dotted lines) as a function of logarithmic time for different magnetic configurations. Lines with color-filled markers are the mean value of edge markers (initial minor radius $\rho>0.5)$ and white-filled markers for the core. Logarithmic ordinate scale highlights the difference in the loss fractions. The line legend is in table 1
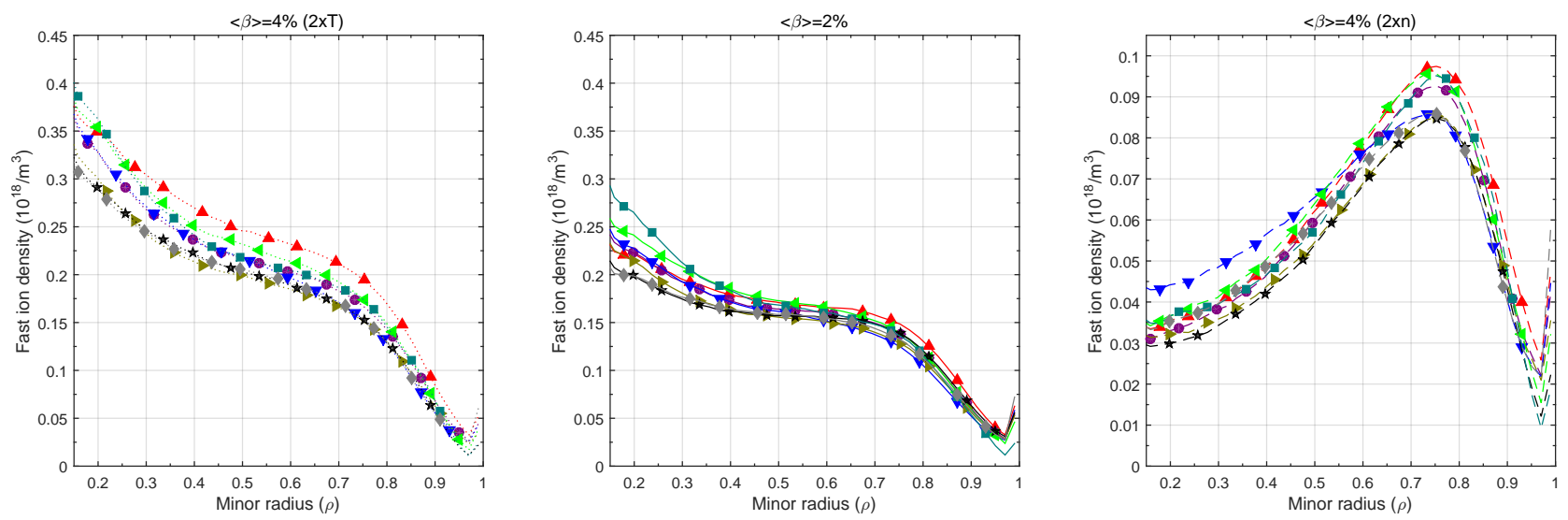

Figure 7. The fast ion density in different magnetic configurations with three different plasma profiles. The profiles are integrals over the whole velocity space (summing over the whole slowing down process). Note that the ordinate scale varies from panel to panel. The line legend is in table 1 

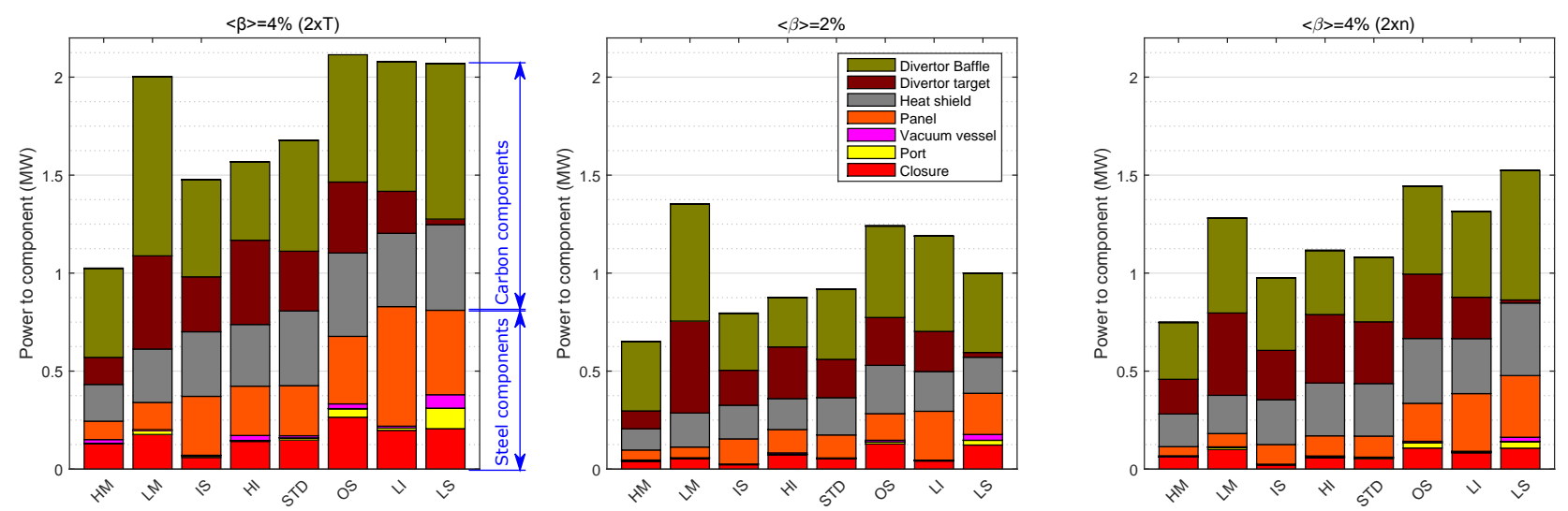

Figure 8. Wall power load results of the magnetic configuration scan. Full color legend is shown in figure 3 The abbreviated names are given in table 1 The injected NBI power was $6.8 \mathrm{MW}$

\subsection{Detailed analysis of loss patterns}

The analysis of the wall loads in different configurations starts by comparing the power deposited to various plasma facing components (figure 8). The high mirror (HM) configuration and the inward shifted (IS) configurations exhibit not only minimal total lost power, but also very favorable distribution of the lost power: the sensitive steel components receive only a small fraction of the total power deposited to the PFCs. The losses to steel in the low mirror (LM) configuration are comparable to HM and IS cases, but high losses to the divertor and baffle suggest the overall fast ion confinement is reduced.

The most intuitive way to study the distribution of heat load on the complex wall is to study it with perspective views such as in figure 1. However, such images can only present a fraction of the whole wall. Therefore, the supplementary material includes a flythrough video of the results of one case, the high statistics run, that is described in detail later in section 3.3 .

For the purpose of analyzing and comparing the overall load patterns, a toroidal-poloidal projection of the wall loads is shown in figure 9 for three configurations of the $\langle\beta\rangle=2 \%$ cases. In all configurations, the hot spots are located mainly on the targets and otherwise between the modular coils. The difference between the magnetic configurations are generally variations in the relative strength of the hot-spots, some of which may be absent in certain configurations. It is informative to compare the toroidal distribution of the loads with toroidal structure of the magnetic field: figure 10 shows how the passing particles (with a large value for the constant of motion $B_{t}=E / \mu=$ energy $/$ magnetic moment) follow the field lines to the targets and the baffle. Losses to steel components are primarily due to particles with $B_{t}$ approximately up to the maximum $|\boldsymbol{B}|$ on axis, presumably corresponding to trapped and helically trapped orbits that are only poorly confined. The load is not toroidally symmetric around module (field period) centre (toroidal angle $=0$ ), which is explained by the stellarator-antisymmetric nature of the dominant guiding centre drift, the grad-B drift.

Further analysis of the particles hitting the wall (figure 11) reveal that each hot spot on the wall is characterized by the reflection magnetic field $\left(B_{t}=\right.$ $E / \mu)$ and particle pitch $\left(v_{\|} / v\right)$ at the wall. Therefore, a certain volume of phase space is responsible for each hot spot and the small absolute value of the pitch indicates that the hot spots are formed by trapped particles that have drifted to the wall in the local magnetic wells.

The heat load to the targets and baffles are caused by passing particles (high $B_{t}$ ) predominantly following the field lines (high absolute values of pitch) to the plasma facing components. The prompt and first-orbit losses are passing particles and are also conducted to the divertor region. Beyond them, there is almost no dependency on the particle energy nor time from ionization to wall hit in the various hot spots. (The energy and time as well as other plots are presented in the supplementary material.) This indicates that a long diffusive process through phasespace is responsible for the hot spots. Due to this diffusion, there are only minor differences between the four studied NBI sources (Q3, Q4, Q7 and Q8) [18.

A further observation can be made as a synthesis of figures 911 . The HM configuration has a strong toroidal magnetic well, which gives rise to relatively large $B_{t}$ variations in each hot spot. Therefore, the spots are quite large and diffuse in HM, while in LM they are smaller and better pronounced. 

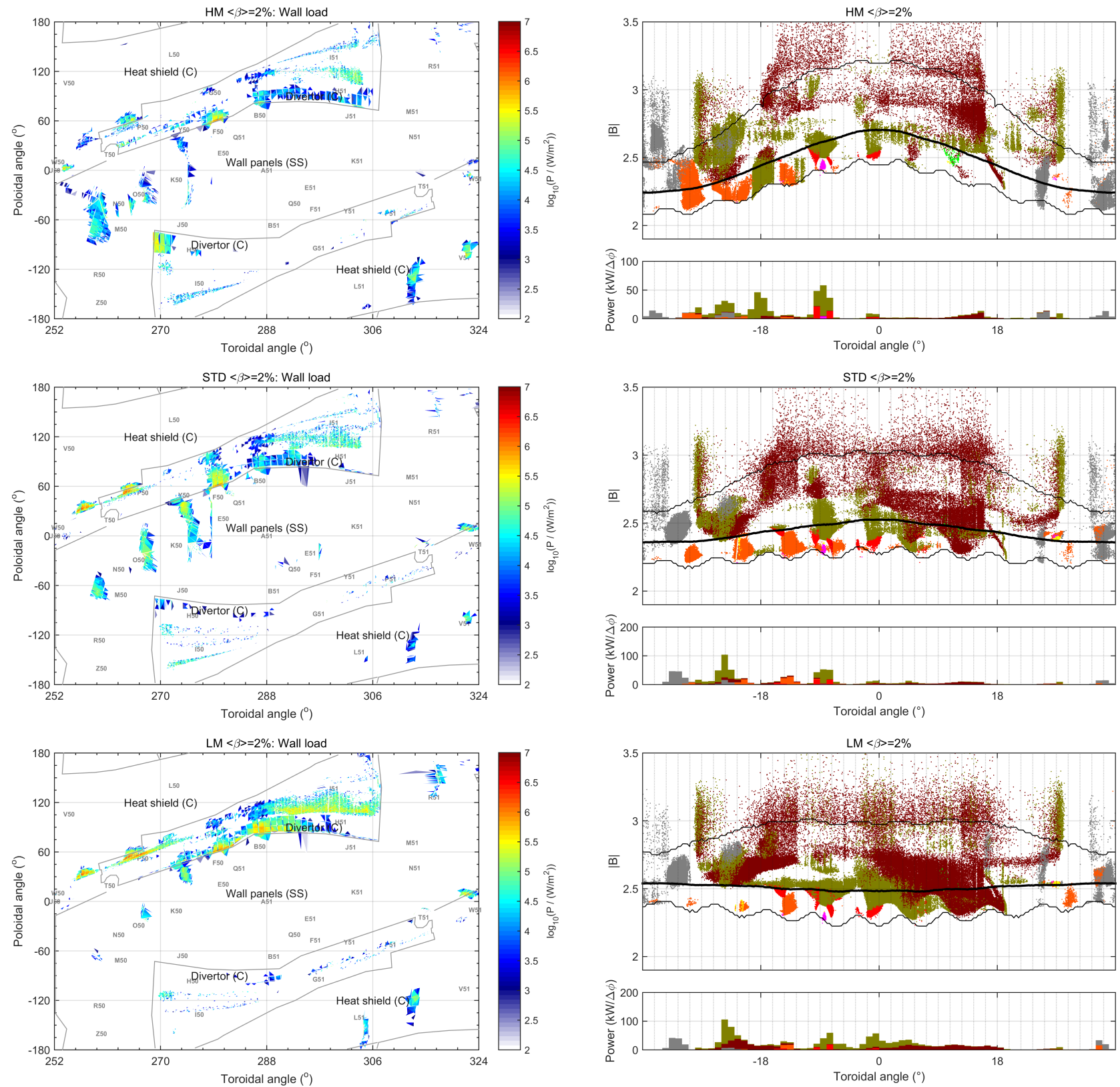

Figure 9. The wall load in the high mirror (HM), standard (STD) and low mirror (LM) configurations in module 5. The prompt losses do not extend to this module. The color indicates the power to the wall $\left(\mathrm{W} / \mathrm{m}^{2}\right)$ on logarithmic scale. This figure is most useful for assessing the spatial distribution of the loads: e.g. the panels seem to be less loaded in LM than in other configurations. However, figure 8 indicates them to be equal in HM and LM. The gray codes indicate port locations and the lines draft the boundaries of PFC regions (divertor, heat shield and panels). The poloidal angle is calculated using $\theta=\operatorname{atan} 2\left(z-z_{0}(\phi), R-R_{0}(\phi)\right)$, where $R_{0}$ and $z_{0}$ are locations of the magnetic axis in the vacuum standard configuration as a function of the toroidal angle $\phi$

Figure 10. The toroidal distribution of wall hits in three different magnetic configurations. Each colorful dot represents a simulated particle hitting the wall, with the color indicating the wall component as given in figure 3 The vertical coordinate indicates the particle reflection magnetic field $B_{t}=E / \mu$. The thick black line is the magnetic field strength at the magnetic axis while the thin ones are the maximal and minimal values within the plasma for each toroidal angle. They clearly visualize the modular coil ripple responsible for most hot spots on the sensitive steel components. The histograms in the lower panels indicate toroidal distribution of power to the various components and are shown to assess the significance of the various contributions. Note the different scale in the HM configuration. Losses to the targets occur also with higher $B_{t}$ than are visible in the figure. The plot is the sum over all five modules. 

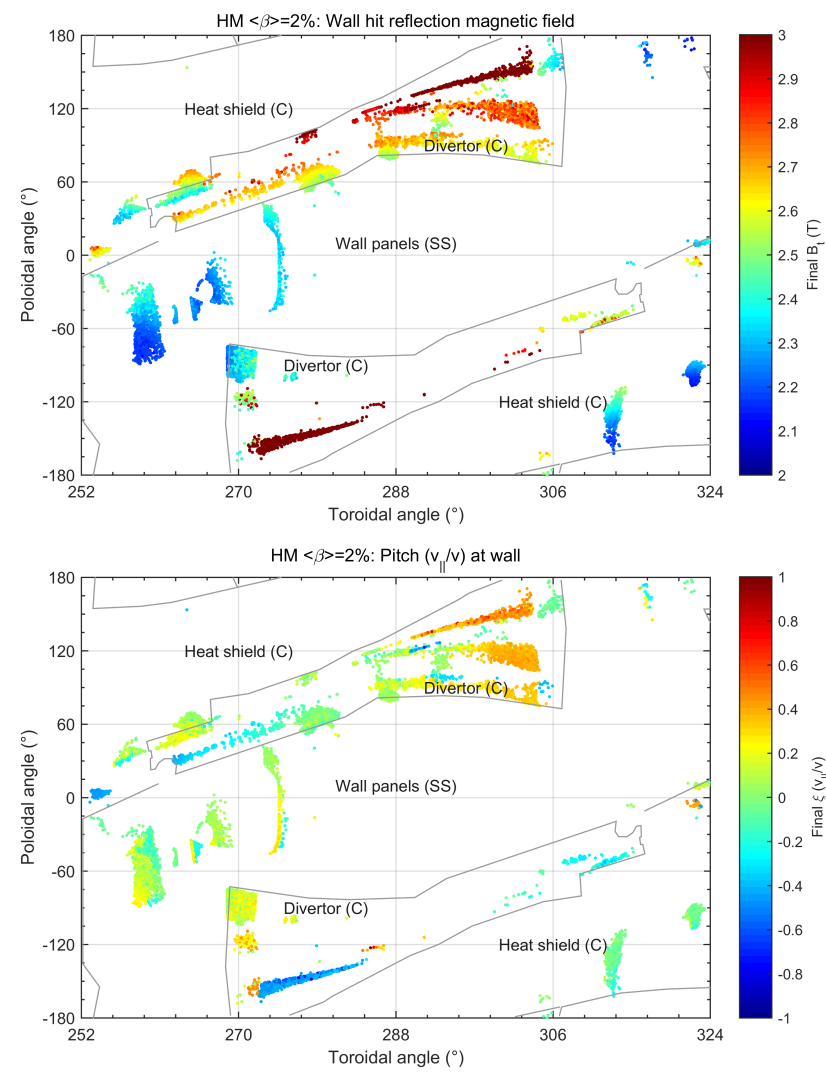

Figure 11. Plasma facing component hits in module 5 for the high mirror configuration at $\langle\beta\rangle=2 \%$. The top panel shows how each hot spot is characterized by distinctive reflection magnetic field $\left(B_{t}\right)$, while the bottom panel indicates the same for particle pitch $\left(v_{\|} / v\right)$. The supplementary material includes further plots of this kind.

The supplementary material includes plots similar to figure 11 for LM and STD configurations.

\subsection{Estimated consequences of the wall load}

The lost NBI ion flux to the PFC has two direct consequences: the component heats up and material is sputtered from the component, making it a source of impurities to the plasma. A preliminary estimate for the sputtered flux of steel by the lost NBI ions is $\sim 10^{18}$ atoms/second, which is similar to the sputtered flux due to the charge exchange ions from the thermal plasma 23. The estimate uses approximated sputtering coefficients for hydrogen irradiation of nickel as a proxy for steel 24. All sputtered atoms were assumed to reach the plasma, which makes this calculation an upper limit or a worst-case estimate for the steel transport. On the other hand, the combined effect of heating and sputtering is not considered at all. The dilution of the plasma due to released impurities is expected to be detrimental to plasma performance, but even doubling the current iron content in the plasma is not expected to cause an intolerable increase in radiation losses. Even in the absolute worst case scenario of radiative collapse of the plasma, no harm to the machine itself is expected.

The heating up of the plasma facing components by the NBI losses limits the maximal safe operating time of the NBI. In the upcoming first NBI studies, projected for 2018, the steel panels and most of the carbon components are not actively cooled [25, 26, 27. On the other hand, the NBI system is internally limited to $7 \mathrm{~s}$ of continuous operation in hydrogen. The critical transient heat load to the components is a complex issue and is still work in progress. It depends i.a. on the cooling and initial temperature of the components. For the current study, we estimate that $2 \mathrm{~s}$ pulse of $1 \mathrm{MW} / \mathrm{m}^{2}$ or $0.1 \mathrm{~s}$ pulse of $5 \mathrm{MW} / \mathrm{m}^{2}$ for the steel panels and $1 \mathrm{~s}$ pulse of $10 \mathrm{MW} / \mathrm{m}^{2}$ for carbon components does not damage the components. However, the value for steel is already an order of magnitude above the design steady state heatflux. Accordingly, these estimates cannot be considered as the conclusive nor an authoritative limit.

A 2D projection of the wall loads gives an overview of the losses, but assessing the actual hot spots is difficult. The illustration in figure 12 disposes of the logarithmic scale and shows the heat load in a "simple" plot. Such illustration calls for increased simulation size due to the unfavorable convergence behaviour of quantities that involve the maximum over a histogram. In this case the histogram is the wall load per wall triangle. It is calculated as a sum of energy from the markers to a wall triangle divided by the area of the triangle. As a consequence, a tiny wall triangle receiving a single marker produces a huge heat load. Such small triangles produce strong noise in the analysis. Hence, finding the maximum heat load requires large simulations. A special simulation, the high statistics run, was performed as part of the commisioning of the Marconi-Fusion supercluster and consisted of $10^{8}$ instead of the normal $10^{6}$ markers. The run was, coincidentally, for the W7-X standard configuration in reversed field direction, with $\langle\beta\rangle=$ $2 \%$, and the radial electric field doubled.

The losses in figure 12 have approximately 5periodic structure arising from the 5 -fold machine periodicity. The deviations are more likely due to the asymmetries in the wall structures than in the localised source of ions. For example, the target geometry is highly symmetric across the 5 modules (field periods), making the prompt losses visible near toroidal angles $25^{\circ}$ and $130^{\circ}$. The peak heat loads are generally uncomfortably high for a full 7 second NBI pulse. The carbon components may be compatible with the heat fluxes for a $1 \mathrm{~s}$ period. The peak power loads to steel components would limit the safe NBI operation 


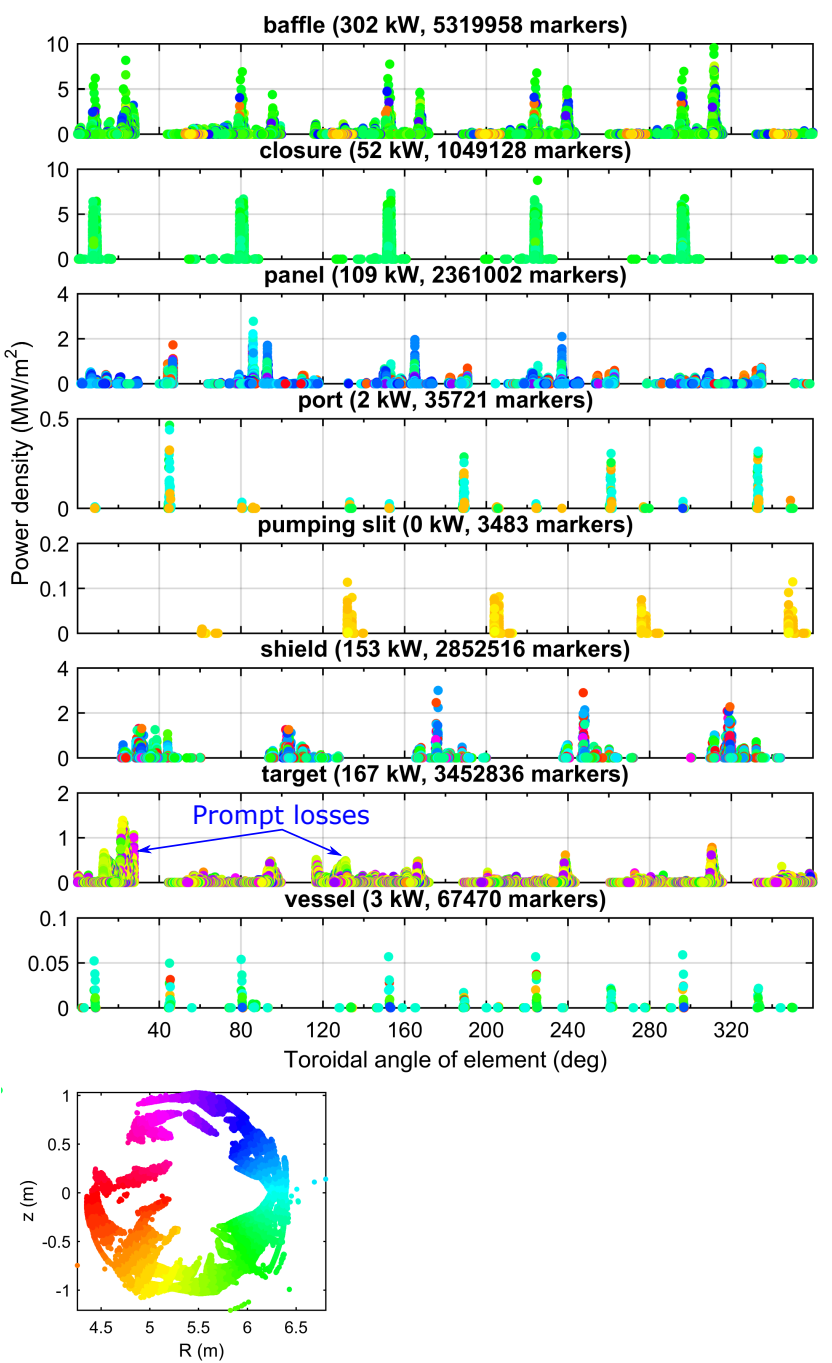

Figure 12. The wall load as a function of toroidal angle separated to wall component types. Each circle in the plot represents a single triangle in the $3 \mathrm{D}$ wall model. The horizontal location of the circles indicates the toroidal location of the triangle and the vertical location indicates the NBI heat load deposited to the triangle. The colour of the circle indicates the poloidal location of the triangle, as shown by the lowest panel. For each component type, the total power and total number of simulation markers deposited is shown. The data is from a unique high-statistics-run of 100,000,000 markers, that was performed for the W7-X standard configuration in reversed field direction, with $\langle\beta\rangle=2 \%$, and the radial electric field doubled.

time with a single NBI source to $\sim 100 \mathrm{~ms}$ for the standard configuration. The initial NBI operations will be performed in magnetic configurations with more benign losses.

To assess the peak heat loads in the normal $10^{6}$ marker simulations, convergence analysis was performed to the $10^{8}$ marker run and to $10^{7}$ and $10^{6}$ marker sub-samples. The peak heat loads for most components are converged with $10^{8}$ markers but not yet with $10^{6}$, where the peak heat loads are uniformly over-estimated. The ensemble of $10^{6}$ markers is enough to trace all strong hot spots and their approximate magnitudes in plots similar to figures 9 and 1. However, the boundaries and weak hotspots are resolved only with $10^{7}-10^{8}$ markers. Fortunately, visual inspection of the wall load illustrations (fig. 90 seems to effectively yield correct estimates for the peak loads already from the $10^{6}$ marker simulation results. Therefore, it is possible to approximately compare also peak heat loads from the poloidal-toroidal maps or perspective views. For example in the $\langle\beta\rangle=2 \%$ high mirror configuration, the hot spots on the panels are few $100 \mathrm{~kW} / \mathrm{m}^{2}$, and the peak loads on carbon component are few $\mathrm{MW} / \mathrm{m}^{2}$. Thus, the loads may allow a few seconds of NBI operation.

The high statistics run enabled detailed study of the internal structure within the hot spots. The simulated hot spots have wide, smooth peaks of particle hits without a focused hot spot in contrast to the experience with e.g. runaway electrons [28. However, the PFCs have leading edges, that may lead to highly localized heating. The most critical single component identified to date is the immersion tubes in the upper F-ports (lower F-ports in reversed field configurations). These 5 immersion tubes contain a number of the cameras that can be used for detection of the hot spots, but the tubes are not included in the wall used in the here presented configuration scan. The tubes are, however, equipped with protective shutters as well as protective steel rings and are visible through the other F-port cameras, which allow close monitoring of these components during initial NBI operations.

As a summary of the configuration scan, the initial NBI operations should be performed in a magnetic configuration with more benign losses than the standard configuration has. From the reference configurations, the high mirror configuration is the best candidate and is expected to allow a few seconds of NBI operation. The role of the plasma profiles is studied next.

\section{Plasma profile scan in high mirror configuration}

The plasma kinetic profiles play a significant role in determining the NBI wall loads, but they are not yet experimentally available. The first neutral beam injection (NBI) heating experiments are planned in the experiments starting in 2018 (dubbed OP1.2b). The 2017 campaign (OP1.2a) has started after the installation of the test divertor. The first experimental campaign (OP1.1) was performed in 2015-2016 with limiter plasmas. Hence, at the time of writing, the W7-X stellarator has been operated only as a limiter 
machine. Unfortunately, the plasma kinetic profiles measured in the limiter operation cannot be expected to be representative of the future diverted plasmas, and the best course of action is to numerically study the effect of different plasma profiles. Accordingly, a set of 18 plasma scenarios in the high mirror configuration was studied: 3 heating options, 2 plasma density profile shapes with 3 scaling factors each.

\subsection{Calculation of the plasma profiles}

It is possible to study numerically arbitrary plasma profiles. However, this scan uses only profiles that are expected to be achievable using the limited options in the real experiment: the temperature can be adjusted by changing the heating, the density by changing the gas puffing, pellets and NBI rates. These changes are non-linearly interlinked and a code is needed to solve the profiles self-consistently. However, in this study the problem was simplified by assuming a single equilibrium in the high mirror configuration when performing the transport calculation with the NTSS code. After the profiles were solved, a new equilibrium with the updated kinetic profiles was calculated for the ASCOT4 simulations (see figure 2).

The NTSS code was utilised to calculate the effect of different rates of ECRH heating (2, 5 or $8 \mathrm{MW})$. The ECRH was modelled in either $\mathrm{X} 2$ or triple pass $\mathrm{O} 2$ mode depending on density. The maximum simulated density of $1.6 \times 10^{20} / \mathrm{m}^{3}$ was chosen to keep the ECRH stray radiation below $1 \mathrm{MW}$ in all modelled cases. The NBI source Q8 was always included as a heating source at $1.7 \mathrm{MW}$ injected power. This needs not to be self-consistent with the ASCOT4 modelling of four sources, because there is no feed-back from the ASCOT4 simulations back to NTSS.

The density profiles are difficult to estimate, hence the shape of the density profiles (peaked or flat) and the density on axis $\left(0.5,1.1\right.$ or $\left.1.6 \times 10^{20} / \mathrm{m}^{3}\right)$ were used as scan parameters. The precise density profile as a function of the normalised minor radius coordinate ( $\rho=r_{\text {eff }} / a$, with $a$ being the effective plasma minor radius of the LCFS) is given by the following formula:

$$
\begin{gathered}
n_{e}(\rho)=m\left[g-h+(1-g+h)\left(1-\rho^{s}\right)^{p}+\right. \\
\left.h\left(1-\exp \left(-\rho^{2} / w^{2}\right)\right)\right] 10^{20} / \mathrm{m}^{3}
\end{gathered}
$$

where $s=4$ gives peaked and $s=12$ a flat density profile. The other parameters are set as follows: $m=0.5 / 1.1 / 1.6 ; g=0.25 ; p=2 ; h=0.025 ; w=0.5$. For the transport calculations, the effective charge was assumed to be $Z_{\text {eff }}=1.5$ with a carbon impurity in hydrogen plasma. For the ASCOT4 simulations the plasma was assumed to be pure proton-electron plasma.

The resulting plasma profiles are presented in figure 13. It is evident that in the two lowest density cases the core of the plasma is in electron root confinement with positive radial electric field $E_{\mathrm{r}}$. The $E_{\mathrm{r}}$ field is increased along with the ECRH heating power also in the edge region where negative $E_{\mathrm{r}}$ implies ion root confinement.

\subsection{NBI ion density and wall loads}

The ASCOT4 simulations of the 18 scenarios were performed similarly to the simulations of the configuration scan. The NBI ionization profiles (figure 14) depend strongly on the density but only weakly on the plasma temperature. At lowest densities up to $\sim 30 \%$ of the neutrals pass through the plasma without ionizing. The heat-load to the beam-dump tiles on the heatshield is high, while still tolerable for short $(\sim 1 \mathrm{~s}) \mathrm{NBI}$ heating pulses. When the density is increased, the ionization profile moves to the edge, but even in the most extreme studied case one-third of the ions are ionized within $\rho<0.5$. The ion losses from the core increase with temperature, which reduces the collisionality and increases the time before the fast ions slow down to thermal energy. This is also corroborated by the loss fractions (figure 15). The initial linear phase on the core (ionisation $\rho<0.5$ ) losses can be fitted with a line in figure 15 . The slope decreases slightly with increasing density, which means the diffusion out takes longer. On the other hand, the loss process start earlier with higher density, most likely due to the ionisation locations trending towards the edge with increasing density. However, the change in slowing down time with the density dominates over the aforementioned effects. The core loss fractions are reduced from $\sim 8.4 \%$ at $0.5 \times 10^{20} / \mathrm{m}^{3}$ to $\sim 4.8 \%$ at $1.1 \times 10^{20} / \mathrm{m}^{3}$, and to $\sim 4.3 \%$, at $1.6 \times 10^{20} / \mathrm{m}^{3}$. The corresponding slowing down time is reduced from $\sim 20 \mathrm{~ms}$ to $\sim 10 \mathrm{~ms}$ and to $\sim 5 \mathrm{~ms}$, respectively. The reported slowing down times are directly calculated from the simulation results as medians of the time it took for the markers to slow down to 1.5 times the local thermal energy or to $1 \mathrm{keV}$ at the edge. The slowing down time is reduced by the contribution of the one-half and one-third energy ions that slow down quickly.

The fast ion density (figure 16) is increased in the regions of high electron temperature via longer slowing down time. This occurs in the core electron root confinement (CERC) part of the plasmas $(\rho<$ $0.3 \cdots 0.5$ for $\left.0.5 \times 10^{20} / \mathrm{m}^{3}\right)$. The positive radial electric field also contributes to increase in fast ion density, but plays only a minor role: doubling the electric field only increased the fast ion density by $\sim 15 \%$. The positive field was actually expected to compete against the beneficial effect of increasing plasma pressure [29] and to reduce the confinement. Finally, the wall load partitioned to components (figure 17) shows that increasing electron heating from 

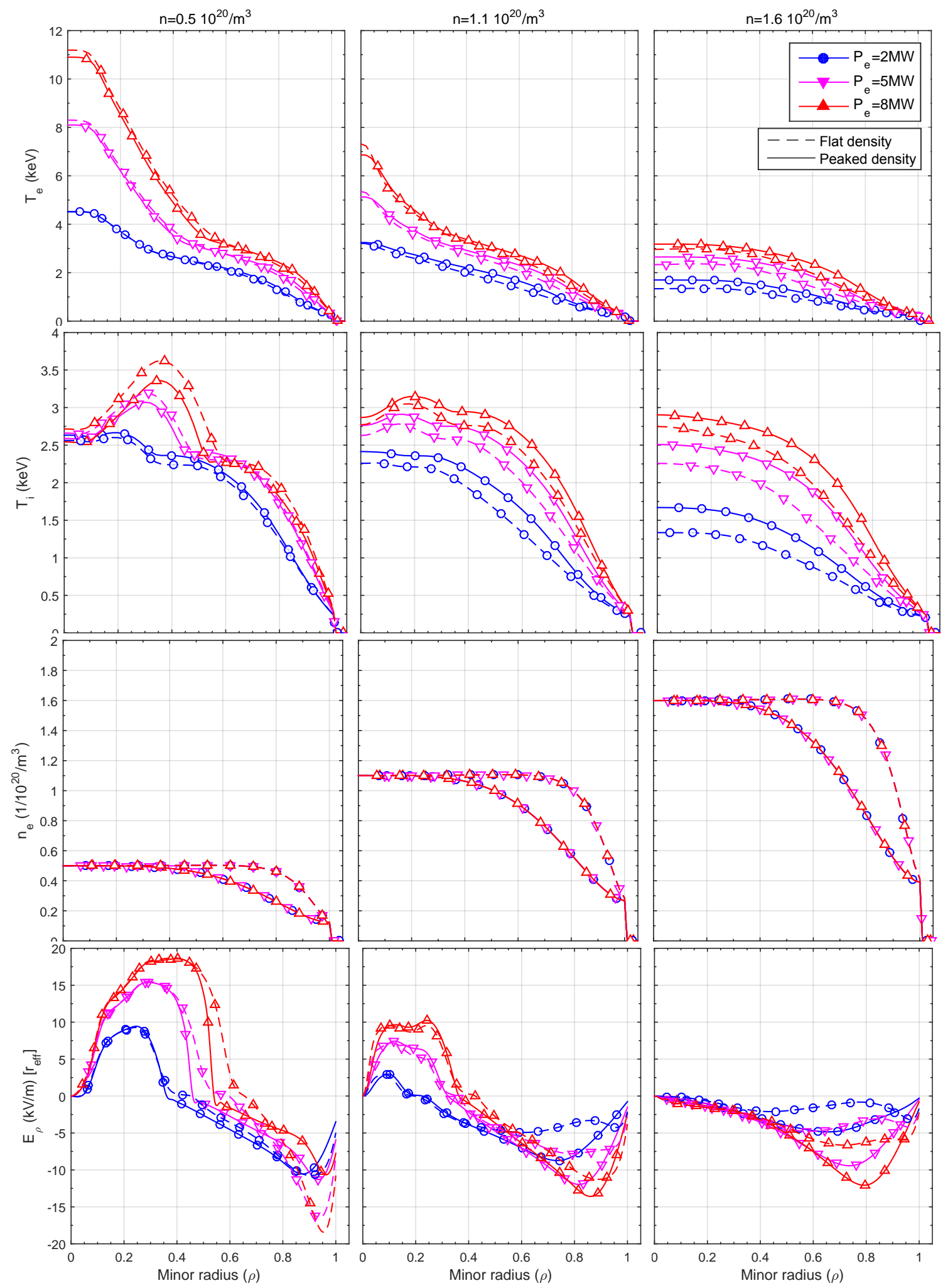

Figure 13. The plasma profiles for the profile scan. These are produced by the transport code and used as input to the fast ion codes. The rows from the top are the electron temperature, the ion temperature, the density and the radial electric field. The columns are the different densities at the magnetic axis, with two profile shape variations each. The core profiles come from purely Neoclassical physics, which gives rise to off-axis peaked ion temperatures in low density cases due to electron-ion temperature decoupling in the hot core. The plasma was assumed to be pure electron-proton plasma. 
$2 \mathrm{MW}$ to $8 \mathrm{MW}$ increases the wall loads by $10-20 \%$, while increasing the density from $0.5 \times 10^{20} / \mathrm{m}^{3}$ to $1.6 \times 10^{20} / \mathrm{m}^{3}$ reduces them by $5-10 \%$. A hypothetical doubling of the radial electric field would reduce the wall loads by $10-20 \%$. 

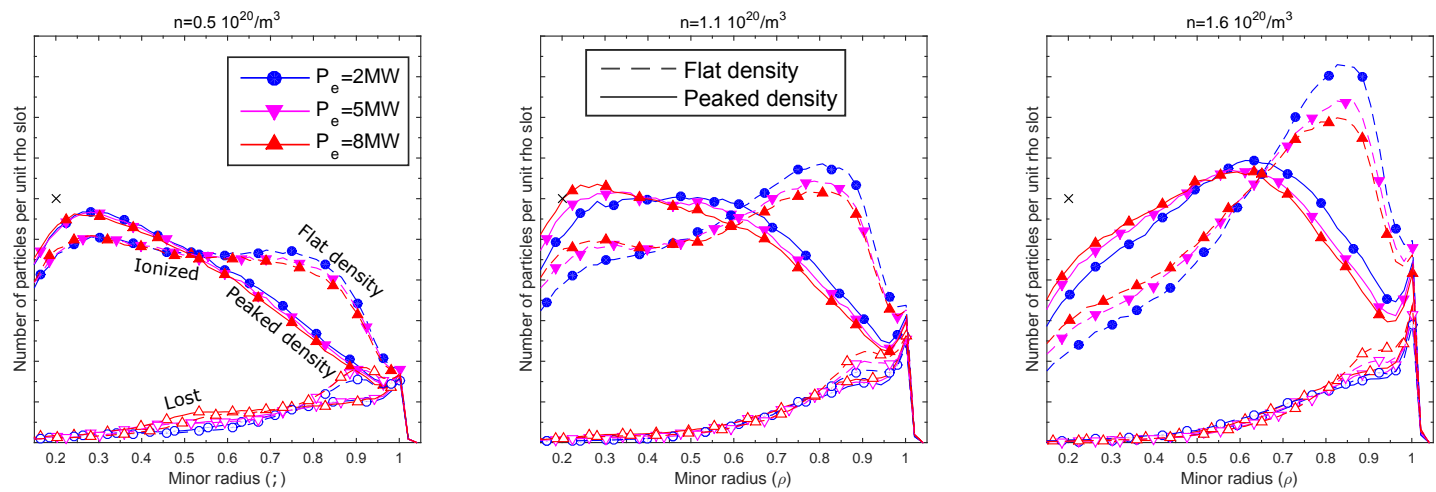

Figure 14. The NBI ionization distribution (filled markers), which was created by simply binning the ionization locations in minor radius. This results in the same but arbitrary ordinate unit for all panels. The peak at the edge is an artifact of the minor radius coordinate: it is only defined within the plasma. Therefore, markers ionized outside the plasma are all collected to the single bin at $\rho=1$. The lines with hollow markers depict the particles that were lost to the wall. The black cross is at same level, both in this figure, and in figure 5
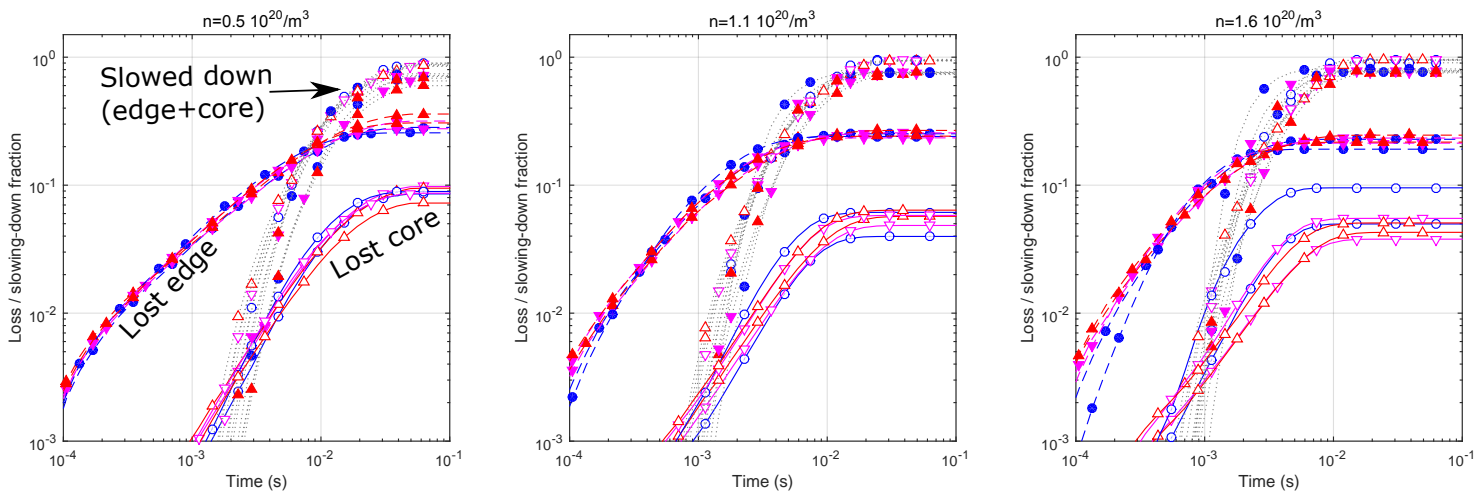

Figure 15. The fraction of particles lost to the wall (colored lines) and slowed down (gray lines) as a function of logarithmic time for different profiles in the profile scan. Lines with filled markers are the mean value of edge markers (initial minor radius $\rho>0.5$ ) and white markers for the core. Logarithmic ordinate scale highlights the difference in the loss fractions.
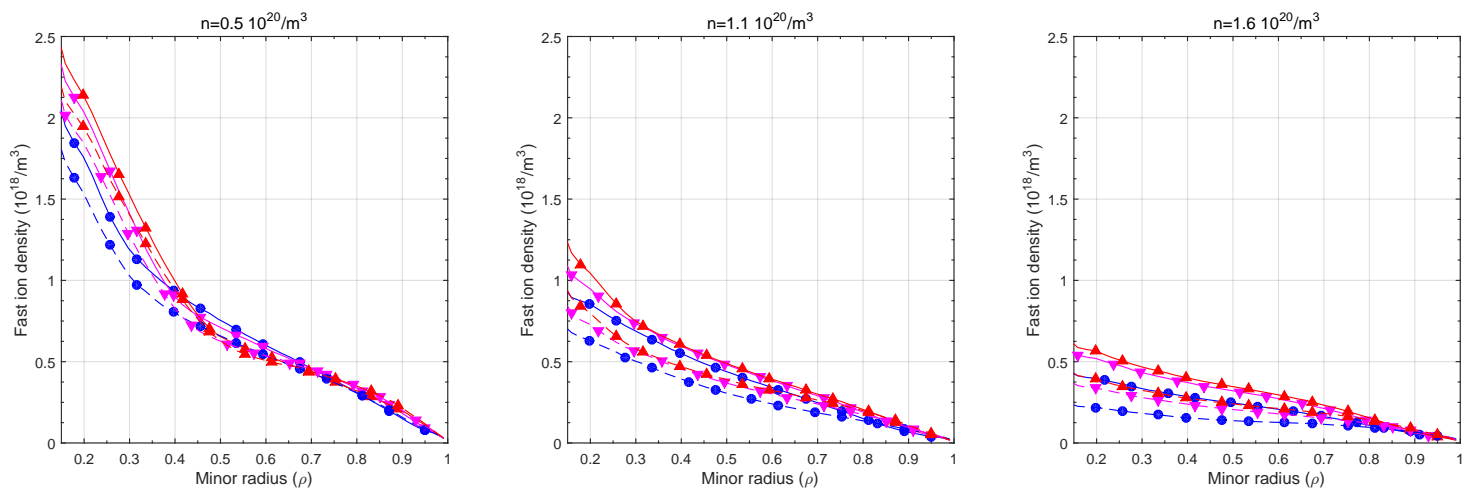

Figure 16. The fast ion density with various plasma profiles. 

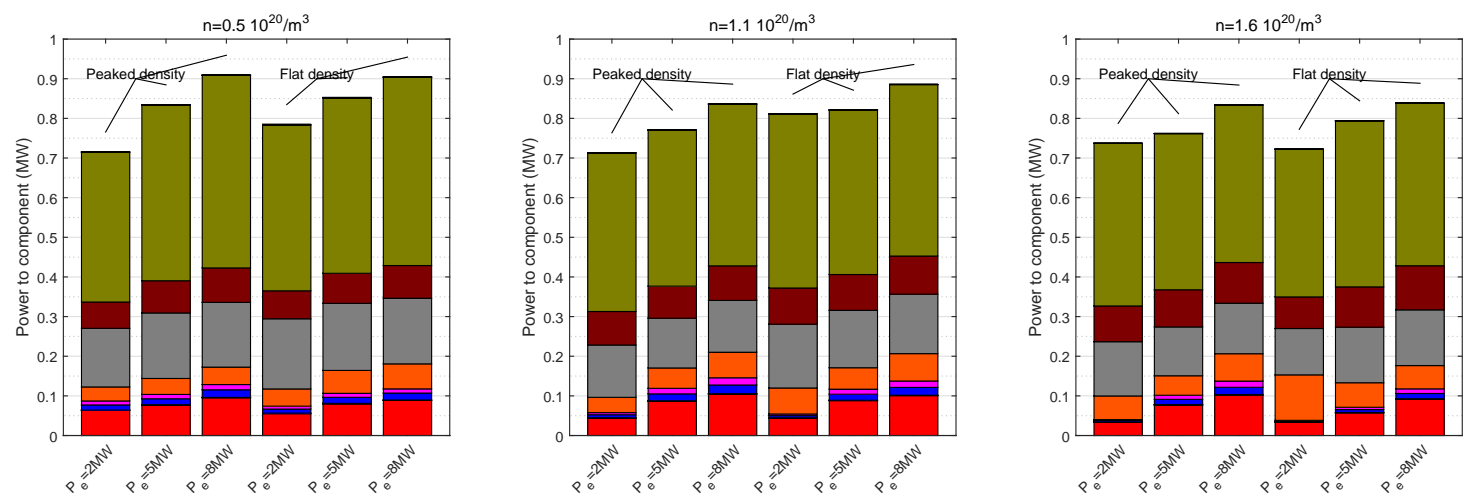

Figure 17. Wall power load results of the profile scan. Full color legend is shown in figure 3

\section{Summary and further work}

This paper showed that the NBI losses will lead to strong heating of the PFCs, in particular certain vulnerable steel components may receive several $\mathrm{MW} / \mathrm{m}^{2}$, in all of the reference magnetic configurations of $\mathrm{W} 7-\mathrm{X}$ and in all simulated plasma profile scenarios. The losses to steel components are due to hot-spots associated with the modular coil ripple. Detailed study of a non-optimal case showed no internal structure in the hot spots and that the wall loads are an order of magnitude or more above steady state design loads. Accordingly, the wall loads are expected to limit the initial maximal safe NBI heating pulse length as well as the magnetic configurations where the NBI injection can be initially used. Loads in the best reference magnetic configuration (high mirror) may limit the NBI operation to few seconds. These estimates will be tested when the NBI systems are commissioned during the operational campaign OP1.2b scheduled for 2018.

This paper further showed that the high mirror configuration has the most benign wall loads of the reference configurations, i.e. the load to the most sensitive wall components is small. These losses can be further reduced by increasing the fast ion collisionality by increasing the density or reducing the temperature. However, the total losses increase with higher collisionality due to the NBI ionisation profile moving towards the edge. At lower collisionality, the losses extend to NBI ions born deeper in the plasma.

There are a number of open issues that remain to be addressed in the future. The following fast ion transport mechanisms were excluded from this study: the charge exchange losses, turbulence and MHD activity. The plasma profiles for all cases are not self-consistently calculated. Rather, self-consistency is relaxed in some cases to favour different parameter scans, even if a self-consistent neoclassical transport calculations could be in principle performed. Such work will be more important in the future when comparing with experimental data. Also, changes in the radial electric field due to the NBI losses were not considered. The impurity transport for sputtered steel was strongly overestimated. (The impurity source was still found to be non-critical.) The used wall model lacked certain sensitive components and the final acceptable transient wall load limits are still under investigation. Many of these issues can be addressed only after the first experiments.

One possibility to further reduce the wall loads would be to use magnetic configurations beyond the reference configurations. The nine reference configurations 21, 22 were the original simultaneous optimization points for the W7-X stellarator coil geometry, so deviations from them are, by default, expected to deteriorate the overall performance of the plasma. However, wall loads were not a direct part of the original optimization process; hence, an optimum for the wall loads may lay elsewhere. By combining the key features of the high mirror configuration and inward shifted configuration, the new ISHM configuration removes all NBI loads from the poloidal closures (and the F-port immersion tubes) with only a minor reduction in confinement. Unfortunately, there is a cost in moving beyond the reference configurations: the mechanical integrity of the coils and divertor operation must be carefully checked, and they may limit the practical usefulness of the new configuration.

Another potential tool to control and mitigate fast ions wall loads are the island control coils, designed for the divertor island control and strike line sweeping. There are two coils per module, and they are capable of both DC and AC operation. A preliminary study showed that control coils operated at stellarator symmetric $\pm 2.5 \mathrm{kA}$ DC only altered fast ions wall loads locally on the targets. A more detailed study investigating the full range of operation of the island control coils for fast ions control remains future work. 
Further future work will include detailed study of the new configuration as well as using experimental plasma profiles from the 2017 operational campaign OP1.2a. Also the detailed wall loads of ICRH ions will be studied. The future work may also include conceptual design of wall armoring against fast ion losses.

\section{Acknowledgments}

The authors would like to thank Joachim Geiger, Tamara Andreeva and Sam Lazerson for help with using VMEC and EXTENDER as well as Otto Asunta for help in setting up the BBNBI NBI model for W7$\mathrm{X}$. The authors would further like to thank Jürgen Baldzuhn, Craig Beidler, Norbert Rust and Paul McNeely for helpful discussions.

This work has been carried out within the framework of the EUROfusion Consortium and has received funding from the Euratom research and training programme 2014-2018 under grant agreement No 633053. The views and opinions expressed herein do not necessarily reflect those of the European Commission. The work was partly funded by Walter Ahlström foundation.

\section{References}

[1] Erckmann V, Hartfuss H J, Kick M, Renner H, Sapper J, Schauer F, Speth E, Wesner F, Wagner F, Wanner M, Weller A and Wobig H 1997 The W7$\mathrm{X}$ project: scientific basis and technical realization 17th IEEE/NPSS Symposium Fusion Engineering (Cat. No.97CH36131) vol 1 pp 40-48 vol.1

[2] McNeely P, Barlak M, Baldzuhn J, Bozhenkov S, Drevlak M, Gawlik G, Heinemann B, Holtum D, Jagielski J, Kairys R, Nocentini R, Riedl R, Rong P, Rust N, Schroeder R, Speth E, Stäbler A, Turos A and Wolf R 2013 Fusion Engineering and Design 881034 - 1037 ISSN 0920-3796 proceedings of the 27th Symposium On Fusion Technology (SOFT-27); Liège, Belgium, September 24-28, 2012 URL http://www.sciencedirect.com/ science/article/pii/S0920379613003001

[3] Helander P 2014 Reports on Progress in Physics $\mathbf{7 7}$ 087001 URL https://doi.org/10.1088/0034-4885/77/ 8/087001

[4] Drevlak M, Beidler C, Helander P, Maassberg H, Turkin $\mathrm{Y}$ and Werner A 2011 Power flux from nbi losses on in-vessel components in W7-X Tech. Rep. PLM:1CDY-T0004 Max-Planck-Institute for Plasma Physics (Internal report)(Internal report)

[5] Drevlak M, Geiger J, Helander P and Turkin Y 2014 Nuclear Fusion 54073002 URL http://stacks.iop. org $/ 0029-5515 / 54 / i=7 / a=073002$

[6] Baldzun J 2012 Fast ion orbit losses in w7-x Tech. Rep. PLM:1-CDY-T0005 Max-Planck-Institute for Plasma Physics (Internal report)

[7] Strumberger E 2000 Nuclear Fusion 401697 URL http: //stacks.iop.org/0029-5515/40/i=10/a=302

[8] Faustin J, Cooper W, Graves J, Pfefferlé D and Geiger J 2016 Nuclear Fusion 56092006 URL http://stacks. iop.org $/ 0029-5515 / 56 / i=9 / a=092006$
[9] Kolesnichenko Y, Lutsenko V, Rudenko T and Helander P 2017 Nuclear Fusion 57066004 URL http://stacks. iop.org/0029-5515/57/i=6/a=066004

[10] Dewar R and Hudson S 1998 Physica D: Nonlinear Phenomena 112 275-280 URL https://doi.org/10. 1016/s0167-2789(97)00216-9

[11] Hirvijoki E, Asunta O, Koskela T, Kurki-Suonio T, Miettunen J, Sipilä S, Snicker A and Äkäslompolo S 2014 Computer Physics Communications 185 13101321 ISSN 0010-4655 (Preprint 1308.1904) URL http://www.sciencedirect.com/science/article/pii/ S0010465514000277

[12] Asunta O, Govenius J, Budny R, Gorelenkova M, Tardini G, Kurki-Suonio T, Salmi A, Sipilä S, the ASDEX Upgrade Team and the JET EFDA Contributors 2015 Computer Physics Communications 188 33-46 ISSN 0010-4655 URL http://www.sciencedirect.com/ science/article/pii/S0010465514003701

[13] Hirshman S P and Whitson J C 1983 Physics of Fluids 26 3553-3568 URL http://scitation.aip.org/content/ aip/journal/pof 1/26/12/10.1063/1.864116

[14] Turkin Y, Beidler C D, Maaßberg H, Murakami S, Tribaldos V and Wakasa A 2011 Physics of Plasmas 18 022505 URL http://scitation.aip.org/content/aip/ journal/pop/18/2/10.1063/1.3553025

[15] Hirshman S P, Shaing K C, van Rij W I, Beasley C O and Crume E C 1986 Physics of Fluids 29 2951-2959 URL https://doi.org/10.1063\%2F1.865495

[16] Marushchenko N, Turkin Y and Maassberg H 2014 Computer Physics Communications 185 165-176 URL https://doi.org/10.1016\%2Fj.cpc.2013.09.002

[17] Drevlak M, Monticello D and Reiman A 2005 Nuclear Fusion 45731 URL http://stacks.iop.org/0029-5515/ $45 / i=7 / a=022$

[18] Rust N, Heinemann B, Mendelevitch B, Peacock A and Smirnow M 2011 Fusion Engineering and Design 86 728 - 731 ISSN 0920-3796 proceedings of the 26th Symposium of Fusion Technology (SOFT-26) URL http://www.sciencedirect.com/science/article/pii/ S0920379611003279

[19] Bozhenkov S, Geiger J, Grahl M, Kißlinger J, Werner $\mathrm{A}$ and Wolf R 2013 Fusion Engineering and Design 88 2997-3006 URL https://doi.org/10.1016/j. fusengdes.2013.07.003

[20] Geiger J, Beidler C D, Feng Y, Maaßberg H, Marushchenko N B and Turkin Y 2015 Plasma Physics and Controlled Fusion 57014004 URL http://stacks.iop.org/ $0741-3335 / 57 / i=1 / a=014004$

[21] Andreeva T 2002 Vacuum magnetic configurations of wendelstein 7-x Tech. Rep. IPP Report III/270 MaxPlanck-Institute for Plasma Physics Garching, Germany URL http://edoc.mpg.de/282107

[22] Andreeva T, Kisslinger J and Wobig H 2002 Problems of Atomic Science and Technology 4 4547 URL http://vant.kipt.kharkov.ua/ARTICLE/VANT_ 2002_4/article_2002_4_45.pdf

[23] Naujoks D 2011 Erosionsabschätzung TZM schrauben Tech. Rep. PLM:1-NBF-T0039 Max-Planck-Institute for Plasma Physics (Internal report)

[24] Behrisch R and Eckstein W 2007 Sputtering by Particle Bombardment: Experiments and Computer Calculations from Threshold to MeV Energies Topics in Applied Physics (Springer Berlin Heidelberg) ISBN 9783540445005

[25] Boscary J, Stadler R, Peacock A, Hurd F, Vorköper A, Mendelevitch B, Cardella A, Pirsch H, Tittes H, Tretter J, Li C, Greuner H and Smirnow M 2011 Fusion Engineering and Design 86572 - 575 ISSN 0920-3796 proceedings of the 26th Symposium of Fusion Technology (SOFT-26) URL http://www.sciencedirect.com/ 
science/article/pii/S0920379610004916

[26] Peacock A, Girlinger A, Vorköper A, Boscary J, Greuner H, Hurd F, Mendelevitch B, Pirsch H, Stadler $\mathrm{R}$ and Zangl G 2011 Fusion Engineering and Design 861706 - 1709 ISSN 0920-3796 proceedings of the 26th Symposium of Fusion Technology (SOFT-26) URL http://www.sciencedirect.com/ science/article/pii/S0920379611004303

[27] Peng X, Bykov V, Köppen M, Ye M, Fellinger J, Peacock A, Smirnow M, Boscary J, Tereshchenko A and Schauer F 2013 Fusion Engineering and Design 881727 - 1730 ISSN 0920-3796 proceedings of the 27th Symposium On Fusion Technology (SOFT-27); Liège, Belgium, September 24-28, 2012 URL http://www.sciencedirect.com/ science/article/pii/S0920379613003505

[28] Lipa M, Martin G, Mitteau R, Basiuk V, Chatelier M, Cordier J and Nygren R 2003 Fusion Engineering and Design 66-68 365-369 ISSN 0920-3796 22nd Symposium on Fusion Technology URL http://www. sciencedirect. com/science/article/pii/S0920379603002175

[29] Kolesnichenko Y I, Lutsenko V V, Tykhyy A V, Weller A, Werner A, Wobig H and Geiger J 2006 Physics of Plasmas 13 072504 URL https://doi.org/10.1063/1. 2210927 